\title{
Genetic Modification of MEOR Bacterium Bacillus licheniformis H Strain by Low Energy Ion Beam Irradiation
}

\author{
Zhang Xiangsheng ${ }^{\mathrm{a}, \mathrm{b}}, \mathrm{Li} \mathrm{Miao}^{\mathrm{c}}$ and Xiang Tingsheng*,b
}

\author{
${ }^{a}$ State Key Lab of Microbial Technology, Shandong University, Shandanan Road, Jinan 250100, P. R. China \\ ${ }^{b}$ Key Laboratory of Exploration Technologies for Oil and Gas Resources, Ministry of Education, Yangtze \\ University, Hubei Jingzhou, 434025, P. R. China \\ ${ }^{c}$ Anhui Agri-Food Safe Key Lab, Anhui Agricultural University, Hefei, 230036, P. R. China
}

\begin{abstract}
The microbial enhanced oil recovery (MEOR) related microorganism, identified as Bacillus licheniformis by physiological and biochemical analyses and $16 \mathrm{~S}$ rDNA sequence analyses, was irradiated with $15 \mathrm{keV} \mathrm{N}^{+}$at the dose of $0 \sim 4 \times 10^{15}$ ions $/ \mathrm{cm}^{2}$. The "saddle model" survival curve showed that the turning point is the dose of $2.5 \times 10^{15}$ ions $/ \mathrm{cm}^{2}$, with the survival rate of $23.3 \%$ and the positive mutation rate of $36.7 \%$. The single clone fermentation experiments show that with the rising of dose treated, the surface tension of the fermentation broth of each group had the tendency to decrease, while the group treated at the dose of $2.5 \times 10^{15}$ ions $/ \mathrm{cm}^{2}$ had the lowest average surface tension. Ultimately five mutants were screened from the colonies and two trains, M314 and M325 with a stable expression after the evaluation of five generations were screened out and ready for next analysis. TLC and FT-IR of fermentation broth components showed that the effective component was cyclic lipopeptide. These research results laid a basis for further study in low energy ion beam induced mutation breeding of petroleum microbes.
\end{abstract}

Keywords: Petroleum microorganism, Ion Irradiation, Mutation breeding, Biosurfactant, MEOR.

\section{INTRODUCTION}

With the emergence of worldwide energy crises, the microbial enhanced oil recovery (MEOR) was focused on in recent years. One of the principle is the production of the biosurfactants by petroleum microbes. Surfactants are amphiphilic molecules consisting of a hydrophilic and a hydrophobic domain, which can decrease interfacial tension of water, oil and rock, emulsify crude oil, and change wettability, etc [1]. Besides MEOR, biosurfactants are widely used in environmental biological remediation, fermentation engineering, cosmetic industry, food industry, etc.

The study of the interaction between low energy ions and organisms started from 1980s in China, and has come into one novel interdisciplinary sciences: Ion Beam Bioengineering Technology (IBBe) [2,3]. The application of IBBe technology on plants, microorganisms and the other organisms includes at least two fiends: the application of stimulation effects low dose ion Irradiation [3-5] and mutation breeding of plants and microorganisms [2,6-8].

As one novel type of mutative source, low energy ( 0 $100 \mathrm{keV}$ ) ion beam has many advantages such as low damage, high mutation rate, wide mutation spectrum and repetition of mutative traits [2, 3, 6-10]. Liu Qingmei et al. applied ion Irradiation on $B$. subtilis and acquired one mutant with enhanced surface activity [8]. Besides this, similar research was rarely reported.

*Address correspondence to this author at the Key Laboratory of Exploration Technologies for Oil and Gas Resources, Ministry of Education, Yangtze University, Hubei Jingzhou, 434025, P. R. China; Tel: +86-53188362416; E-mail: xiangshengzh@hotmail.com
Besides Agrobacterium tumefaciens, another biosurfactant producing strain named strain $\mathrm{H}$ was isolated by the authors from Dagang Oilfield [11]. In this study, $\mathrm{N}^{+}$ion Irradiation was applied to mutate and screen the high-yield biosurfactant mutants. The effects of ion Irradiation dose on survival rate and mutation rate were studied and two mutants were screened out, which laid a solid basis for further mutation and screening.

\section{MATERIALS AND METHODS}

\subsection{Identification of $H$ Strain}

\subsubsection{Culture Media}

Seed media: beef-peptone medium. When plate or slant was made, $15.0 \mathrm{~g} / \mathrm{L}$ agar was added.

Biosurfactant-producing media (g/L): $\quad \mathrm{KH}_{2} \mathrm{PO}_{4} \quad$ 2.7; $\mathrm{K}_{2} \mathrm{HPO}_{4} 13.1, \mathrm{NaCl} ; 5.0, \mathrm{KNO}_{3} 1.0, \mathrm{MgSO}_{4} 1.0, \mathrm{NH}_{4} \mathrm{Cl} 1.0$, Sugar 10, Yeast extract 0.5, pH 7.0. When plates were made, $15.0 \mathrm{~g} / \mathrm{L}$ agar was added [11].

Blood agar media: beef-peptone medium plus 5\% defibered sheep blood, according to [12].

The Biosurfactant-producing media were sterilized by $115^{\circ} \mathrm{C}$ and the other media by $121^{\circ} \mathrm{C}$ for $20 \mathrm{~min}$.

\subsection{Ion Irradiation}

Ion Irradiation was conducted in Institute of Plasma Physics, Chinese Academy of Sciences with specialized Ion Beam Bioengineering Device (IBBe Device) $[2,6]$.

$0.1 \mathrm{~mL}$ seed culture from slant tube added with $10 \mathrm{~mL}$ sterile distilled water was spread on the Petri plate. Then it was implanted with $15 \mathrm{keV}$ nitrogen ion at the doses of 0.5 , 
$1.0,2.0,2.5,3.0$ and $4.0 \times 10^{15}$ ions $/ \mathrm{cm}^{2}$ under dry and vacuum conditions. Each pulse contained $2.5 \times 10^{13}$ ions $/ \mathrm{cm}^{2}$. The time interval was set to be 5 s after every 30 pulses. At the same time, in order to estimate the effects of vacuum on mutation, the plate which was placed in the small target chamber but not implanted was set as controls. The Irradiation was repeated for three times as independent repeats.

\subsection{Determination of Survival Rate}

The control and implanted samples were washed with $1.5 \mathrm{~mL} 0.86 \% \mathrm{NaCl}$ solutions. $0.1 \mathrm{~mL}$ appropriately diluted bacteria solution of treated and control groups was spread for blood agar media (the composition seeing 2.2). After incubation at $28{ }^{\circ} \mathrm{C}$ for $3 \mathrm{~d}$, the number of colonies which were counted to determine the survival rate.

\subsection{Measurement of Broth Surface Tensions}

30 colonies of control and ion implanted groups were sampled randomly and inoculated to slant culture, cultured at $28{ }^{\circ} \mathrm{C}$ for $24 \mathrm{~h}$, and stored at $4{ }^{\circ} \mathrm{C}$. Meanwhile the colonies were inoculated to $10 \mathrm{~mL}$ beef-peptone liquid media, cultured for $3 \mathrm{~d}$ at $28{ }^{\circ} \mathrm{C}$ with the rotating seed of 120rpm. 0.1 $\mathrm{mL}$ fermentation broth was transferred to $10 \mathrm{~mL}$ biosurfactant-producing liquid media, culture under the same conditions. The surface tension of the culture broth supernatant (centrifuged $4000 \mathrm{r} \cdot \mathrm{min}^{-1}$ for $20 \mathrm{~min}$ ) was measured with BSY-1 surface tension instrument [11], with the deioned water, blank beef-peptone liquid media and biosurfactantproducing liquid media as controls.

\subsection{Mutation Rate Analysis}

The colony that has decreased more than $10 \%$ in the surface tension of the fermentation broth than the average value of the control group was considered positive mutant and that increased more than $10 \%$ was considered negative mutant. Then the mutation rates were calculated as literature [7].

\subsection{Mutant Stability Analysis}

Five primarily selected mutants were cultured by the tube $\rightarrow$ plate $\rightarrow$ shaking flask $\rightarrow$ tube...procedures with five continuous generations. The fermentation broths of each generation were determined as the former protocols.

\subsection{Thin-Layer Chromatography (TLC) Analysis of Fermentation Broth}

The supernatant $\left(8000 \mathrm{r} \cdot \mathrm{min}^{-1}, 20 \mathrm{~min}\right)$ of the biosurfactant-producing fermentation broth was extracted by chloroform: methanol $=2: 1(\mathrm{~V} / \mathrm{V})$ and submitted to TLC analysis with the chloroform: methanol: water $=65: 15: 2(\mathrm{~V} / \mathrm{V} / \mathrm{V})$ solution as a developing solvent. The chromogenic reagent contained: (1) phenol-sulfuric acid reagent: $3 \mathrm{~g}$ phenol and five $\mathrm{mL}$ concentrated sulfuric acid were dissolved into 95 $\mathrm{mL}$ ethanol, on which the glycolipids show brown dot blot; (2) ammonium molybdate - perchloric acid chromogenic reagent for phospholipids; (3) ninhydrin reagent for lipopeptides which will show red dot blot [11].

\subsection{Fourier Transform Infrared Spectrum (FT-IR) Analysis Fermentation Broth}

Concentrated hydrochloric acid $(\mathrm{HCl})$ was added to the supernatant $\left(10000 \mathrm{r} \cdot \mathrm{min}^{-1}, 15 \mathrm{~min}\right)$ of the biosurfactantproducing fermentation broth to adjust the $\mathrm{pH}$ to 2.0 , put at $4{ }^{\circ} \mathrm{C}$ for one night, centrifuged (10000 r $\left.\cdot \mathrm{min}^{-1}, 30 \mathrm{~min}\right)$ to acquire the precipitate, washed twice with $\mathrm{pH} 2.0$ hydrochlo- ric acid, dissolved into $\mathrm{NaOH}$ to adjust the $\mathrm{pH}$ to 7.0, frozen dried to acquire crude sample. When purified, the crude sample was extracted again in dichloromethane, evaporated under reduced-pressure by rotary evaporator, dissolved again in $\mathrm{NaOH}$ to adjust the $\mathrm{pH}$ to 7.0 to come into vesicle-like liquid, and filtered. The filter liquor was added by $\mathrm{HCl}$ to adjust the $\mathrm{pH}$ to 2.0 , and centrifuged to get the precipitate. The pale yellow precipitate was dried under vacuum to get the purified sample which was ultimately submitted to FT-IR analysis by the KBr squash method [11].

\section{RESULTS}

\subsection{Identification of Strain $\mathbf{H}$}

Strain $\mathrm{H}$ showed flower-alike colonies on beef-peptone plates, but in the beginning appeared round colonies (Fig. 1). It was identified as $B$. licheniformis with the microscopic examination, biochemical analysis (Table 1) and 16s rDNA analysis.

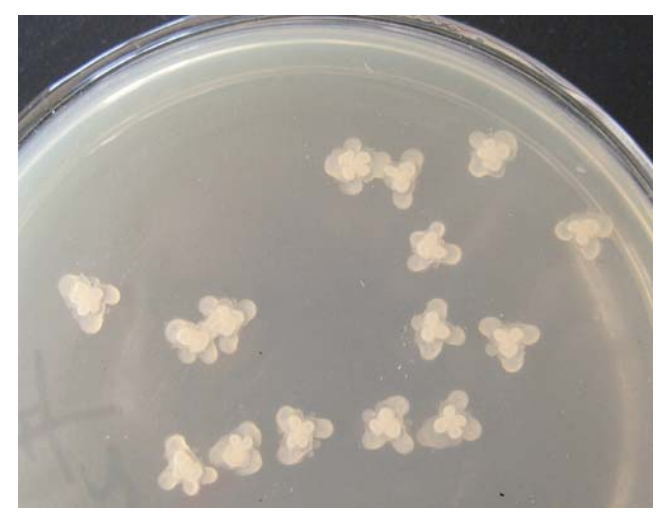

Fig. (1). The colony formed on beef-peptone medium.

\subsection{Effect of Irradiation Dose on the Survival Rate}

It could be seen from Fig. (2) that the survival rate decreased sharply with the rising of dose, but increased a little at the dose of $2.5 \times 10^{15} \mathrm{ions} / \mathrm{cm}^{2}$, then decreased again. And this accorded with the "saddle curve" characteristics. According to the rules of low-energy ion beam induced mutation breeding, the "inflexion point" $\left(2.5 \times 10^{15}\right.$ ions $\left./ \mathrm{cm}^{2}\right)$ was commonly regarded as the most appropriate dose.

Data were pooled from three independent experiments. Error bars indicate SDs of mean values (ANOVA analysis).

\subsection{Effects of $\mathrm{N}^{+}$Ion Irradiation Dose on Surface Tension of Fermentation Broth}

As shown in Fig. (3), with an increase in $\mathrm{N}^{+}$Irradiation dose, the surface tensions showed a tendency to decrease. But the average values of each group had no significant difference $(\mathrm{P}>0.05)$, and this showed that mutation caused by $\mathrm{N}^{+}$Irradiation could be both positive and negative. But the $2.5 \times 10^{15}$ ions $/ \mathrm{cm}^{2}$ group had the lowest average value. And, moreover, this group also had lowest maximum and minimum values. Five colonies with the lowest surface tension were picked out and prepared for genetic stability analysis.

\subsection{Effect of $\mathbf{N}^{+}$Dose on Mutation Rate}

As shown in Fig. (4), the positive and negative mutation rate usually increased with the rising doses. The highest 
Table 1. Cultural and Biochemical Characteristic Features of H Strain

\begin{tabular}{|c|c|c|c|}
\hline Test & Results & Test & Results \\
\hline \hline Form & Rods & Oxidation / fermentation & fermentation \\
\hline Gram's Stain & + & Acid production from glucose & + \\
\hline Endospore formation & + & Gas production from glucose & - \\
\hline Agar cultures & $\begin{array}{c}\text { Flower-like, Entire, Convex smooth or rough, } \\
\text { hair-like edge after long-time culture }\end{array}$ & Voges proskauer test & + \\
\hline Relation to free oxygen & Aerobic & Methyl red test & + \\
\hline Pigment producing & - & Catalast test & + \\
\hline Nitrate reduction test & + & Statin liquefaction hydrolysis & $+($ slow) \\
\hline Indole test & - & Egg yolk agar culture & + \\
\hline Citrate utilization test & + & + \\
\hline
\end{tabular}

+ positive - negative.

positive mutation rate was obtained at $2.5 \times 10^{15}$ ions $/ \mathrm{cm}^{2}$ and considered the most appropriate dose for mutation breeding. At this dose, the positive mutation rate was $36.7 \%$ and the

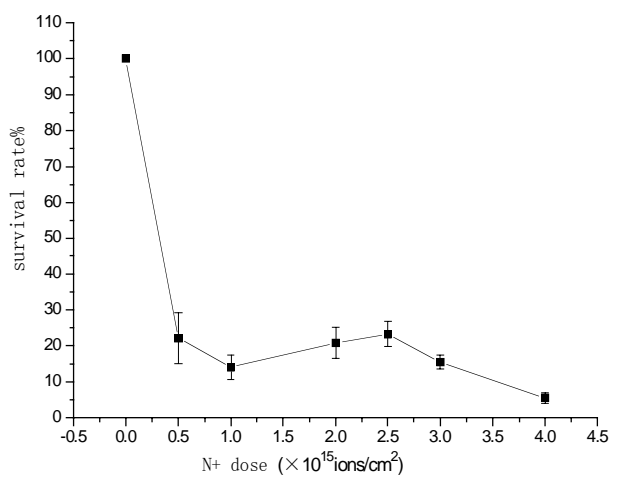

Fig. (2). Effect of $\mathrm{N}^{+}$Irradiation dose on survival rate.

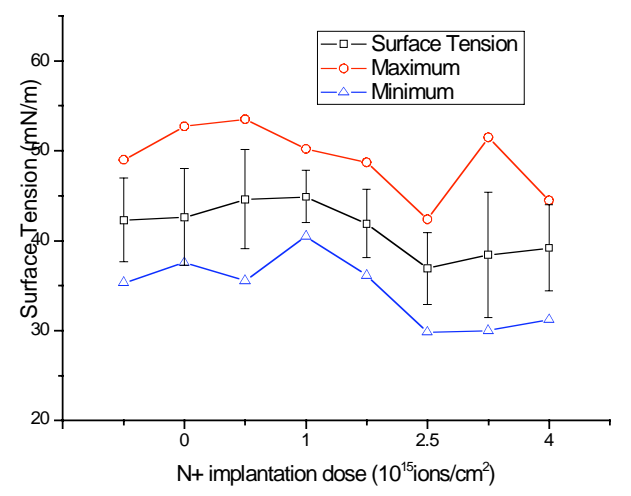

Fig. (3). Effects of $\mathrm{N}^{+}$ion Irradiation dose on surface tension.

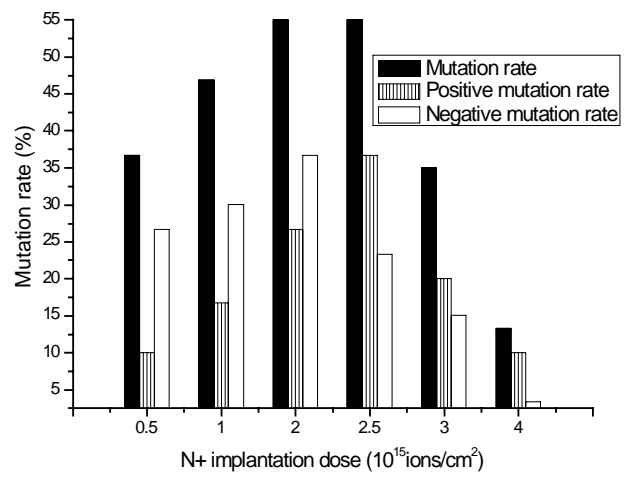

Fig. (4). Effects of $\mathrm{N}^{+}$Irradiation dose on mutation rate. negative mutation rate was $23.3 \%$. When the dose increased above $2.5 \times 10^{15}$ ions $/ \mathrm{cm}^{2}$, both the positive and negative mutation rates decreased simultaneously.

\subsection{Genetic Stability Analysis of Mutants}

The genetic stability of five potential mutants with lowest broth surface tension was analyzed by generation transfer test. The results of the generation transfer are shown in Table 2. The mutant M314 and M325 performed the best, with the surface tension of about $30 \mathrm{mN} / \mathrm{m}$, decreased by $29.9 \%$ and $28.6 \%$, compared to the wild strain and very stable in the generation transfer experiment.

\subsection{Identification of Biosurfactant Produced by $\mathbf{H}$ Strain}

The TLC analysis of fermentation broth showed no color on thin layer when ninhydrin reagent was added. But when the layer was put into sealed bottle filled with $\mathrm{HCl}$ solution at $105{ }^{\circ} \mathrm{C}$ to be hydrolyzed in situ, the ninhydrin could give red dot blot. The other chromogenic reagents test showed no color. The results showed that the biosurfactant didn't have free amino groups but could release free amino groups when hydrolyzed by acid. It could be concluded that the biosurfactant molecular might have the ringed peptide bond.

The supernatant of the fermentation broth appeared white fluffy precipitate when acidized by $\mathrm{HCl}$ and put at $4{ }^{\circ} \mathrm{C}$ for one night. After purification, the biosurfactant sample was submitted to FT-IR analysis. It was found that $3305.83 \mathrm{~cm}^{-1}$ was the N-H stretching vibration caused by hydrogen bonds between molecular chains, $3073.17 \mathrm{~cm}^{-1}$ was $\mathrm{N}-\mathrm{H}$ stretching spectral band caused by intra-molecular hydrogen bonds of $\mathrm{N}-\mathrm{H}$ groups, $1362.72 \mathrm{~cm}^{-1}$ and $1448.57 \mathrm{~cm}^{-1}$ were absorption spectral bands of amide bonds. This suggested that the biosurfactant molecular might have peptide bonds and the hydrophilic moiety should be peptide chains. It could also be seen that $2285.13 \sim 2856.48 \mathrm{~cm}^{-1}$ was $\mathrm{C}-\mathrm{H}$ stretching vibration of fatty acid family, $1623.19 \mathrm{~cm}^{-1}$ was carbonyl group absorption of the lactone. Then it can be concluded that the hydrophobic moiety should be one fatty acid half-molecular. The analysis results of the biosurfactant accorded the characteristics of cyclic lipopeptide suggesting that the biosurfactant should be one kind of cyclic lipopeptide and it is the cyclic lipopeptide that caused the lowered surface tension of fermentation broth.

\section{DISCUSSIONS}

Ion Beam Microbial Engineering is on one of the most important branches of Ion Beam Bioengineering $[3,6]$, and 
Table 2. Generation Transfer Experiments of Five Potential Mutants

\begin{tabular}{|c|c|c|c|c|c|c|}
\hline Generations & M314 & M325 & M410 & M421 & M510 & Original Strain \\
\hline \hline 1 & 34.6 & 34.5 & 35.0 & 35.2 & 33.5 & 45.9 \\
\hline 2 & 32.2 & 32.9 & 34.5 & 34.4 & 35.2 & 46.5 \\
\hline 3 & 28.9 & 31.2 & 32.6 & 32.8 & 30.7 & 43.1 \\
\hline 4 & 30.0 & 32.4 & 34.9 & 31.9 & 32.3 & 43.8 \\
\hline 5 & 31.9 & 29.0 & 32.1 & 35.6 & 34.7 & 42.5 \\
\hline Average & 31.4 & 32.0 & 33.8 & 34.0 & 27.4 & 0.0 \\
\hline Decrease by \% & 29.9 & 28.6 & 24.5 & 24.2 & 4.8 \\
\hline
\end{tabular}

the IBBe technology has been widely applied to industrial microorganisms. Yet very few studies were carried out in petroleum microorganisms. The MEOR is the use of microbes in petroleum reservoirs to enhance the amount of oil that can be produced. An increase in oil recovery by just $1 \%$ will potentially have a much larger benefit for the oil selfsufficiency than the discovery of small satellite fields around proven oil accumulations. The processes that facilitate oil production are complex and may involve multiple biochemical processes. Microbial biomass or biopolymers may plug high permeability zones and lead to a redirection of the water flood, produce surfactants which lead to increased mobilization of residual oil, increase gas pressure by the production of carbon dioxide or reduce the oil viscosity due to digestion of large molecules. The processes behind MEOR are still poorly understood. In this study, the mutation breeding of biosurfactant producing petroleum microorganism B. licheniformis strain $\mathrm{H}$ was successfully conducted and this reaffirmed the efficiency of low energy ion Irradiation in mutation breeding.

In our work, the survival rate curve showed "saddle curve" characteristics, which accorded with the previous literatures $[7,13,14]$. This usually attributed to the result of the comprehensive effects of energy deposition, momentum transfer and mass deposition [3,4,9]. The dose-depended survival rate and mutation rate measurement are the primary means to determinate the optimal Irradiation parameters. It could also be concluded that the irradiation dose of $2.5 \times 10^{15}$ ions $/ \mathrm{cm}^{2}$, which was the "inflexion point" at the survival rate curve, was optimal for mutation breeding.

B. licheniformis strain $\mathrm{H}$ showed ability by decreased surface tension of fermentation broth greatly compared to blank broth. But unlike the other biosurfactant-producing microorganisms such as $B$. Subtilis [8], strain $\mathrm{H}$ failed to produce hemolytic circles on blood agar plates. This showed that the biosurfactant produced by strain $\mathrm{H}$ was not surfactin alike small molecular ones, but large molecular emulsifier. By a series of analysis, the emulsifier might be cyclic depsipeptide.

Yakimov M.M et al. (1997) reported that B. licheniformis BNP29 in the study met all of the criteria when injected into low-permeability cores and, in addition, was able to produce a broad spectrum of metabolites, including solvents, acids, gases and surfactant, whose production can be important in the oil recovery process [15]. It could be concluded that $B$. licheniformis is a kind of bacterium with great potential application for MEOR. The next step is to make further evaluation and screening in the ion implanted population to acquire more outstanding mutants.

\section{ACKNOWLEDGEMENTS}

This work was supported by the Research Project of Department of Education of Hubei Province, China (No. Q20081201), Open Project of Key Laboratory of Microbial Technology of Shandong University and National Science Foundation of China (No. 10905035).

\section{REFERENCES}

[1] Zhao X, Jiang B. Brief Review on MEOR Technology. Petrol Sci 2004; 1: 17-23.

[2] Feng H, Yu Z, Chu P. Ion implantation of organisms. Mater Sci Eng R 2006; 54: 49-120.

[3] Yu Z. Introduction to Ion Beam Biotechnology (translated by Yu L D, Thiraphat V and Ian Brown). New York: Springer Science+ Business Media, Inc. 2006: pp. 145-9.

[4] Song Z, Liang Y, Zhang X, et al. Biological effects of low energy ion beam implantation on plant. Curr Top Plant Biol 2006; 7: 7584.

[5] Zhang X, Xiong T. Improving the Salt Tolerance of Glycyrrhiza uralensis with $\mathrm{N}^{+}$Ion Irradiation. Russ J Plant Physiol 2008; 55: 381-6.

[6] Gu S, Li S, Feng H, et al. A novel approach to microbial breeding-low-energy ion implantation. Appl Microbiol Biot 2008; 78: 201-9.

[7] Gu S, Yao J, Yuan Q, et al. A novel approach for improving the productivity of ubiquinone-10 producing strain by low-energy ion beam irradiation. Appl Microbiol Biot 2006; 72: 456-61.

[8] Liu Q, Yuan H, Wang J, et al. A Mutant of Bacillus subtilis with High-producing Surfactin by Ion Beam Implantation. Plasma Sci Technol 2006; 8: 491-6.

[9] Yu Z. Study on the interaction of low-energy ions with organisms. Suf Coat Tech 1997; 201: 8006-13.

[10] Zhang X, Li Y, Wu Y, et al. Mutation breeding of an organic phosphorus solubilizing bacteria B3 by low energy ion beam implantation. Plasma Sci Technol 2008; 10: 645-50.

[11] She Y, Zhang F, Zhou L, et al. Bio-surfactantants producing endogenous bacteria in Danggang Kongdian Oil Field. J Oil Gas Tech 2005; 27: 88-90(In Chinese).

[12] Wang Y. Production of Rhamnolipid Bio-surfactantsants Separation of Pseudomonas. Biaomian Jishu(Surf Tech) 2006; 35: 69-70.

[13] Liu Q, Yao J, Pan R, et al. A mutant strain of a surfactantproducing bacterium with increased emulsification activity. Plasma Sci Technol 2005; 7: 2885-92.

[14] Liu J, Liu M, Wang J, et al. Enhancement of the Gibberella zeae growth inhibitory lipopeptides from a Bacillus subtilis mutant by ion beam implantation. Appl Microbiol Biot 2005; 69: 223-28.

[15] Yakimov M, Amro M, Bock M, et al. The potential of Bacillus licheniformis strains for in situ enhanced oil recovery. J Petrol Sci Eng 1997; 18: 147-60.

(C) Xiangsheng et al.; Licensee Bentham Open.

This is an open access article licensed under the terms of the Creative Commons Attribution Non-Commercial License (http://creativecommons.org/licenses/by-nc/3.0/) which permits unrestricted, non-commercial use, distribution and reproduction in any medium, provided the work is properly cited. 